\title{
Constraints on Body Movement during Visual Development Affect Behavior of Evolutionary Robots
}

\author{
Mototaka Suzuki, Dario Floreano \\ Laboratory of Intelligent Systems \\ Swiss Federal Institute of Technology (EPFL) \\ CH-1015 Lausanne, Switzerland \\ E-mail: Name.Surname@epfl.ch
}

\author{
Ezequiel A. Di Paolo \\ Centre for Computational Neuroscience and Robotics \\ University of Sussex \\ Brighton, BN19QH, UK \\ E-mail: ezequiel@sussex.ac.uk
}

\begin{abstract}
We explore the role of active body movement in the developmental process of the visual system. Receptive fields of an evolved mobile robot are developed during active or passive movement with the generalized Hebbian algorithm [15]. In accordance to experimental observations of kitten, we show that the receptive fields and behavior of the robot developed under active condition significantly differ from those developed under passive condition. A possible explanation of this difference is derived by correlating receptive field formation and behavioral performance in the two conditions.
\end{abstract}

\section{INTRODUCTION}

Held and Hein have shown that the correlation entailed in the sensory feedback accompanying movement plays a vital role in perceptual adaptation [7]. The authors devised an apparatus in which the gross movements of a kitten moving almost freely (active kitten) were transmitted to a second kitten that was carried in a gondola (passive kitten). Kittens had been previously raised in the dark. After an average of 30 hours in the apparatus the active kitten showed normal behavior in several visually guided tasks, such as paw extension on approaching horizontal surface from above and blinking at object put in front of their eyes, while the passive one failed. The authors concluded that visual stimulation correlated with movement was necessary for the development of the visual control of behavior. However, it is still not clear how the active body movement of the kitten enabled it to develop such visually guided behaviors.

The characteristics of biological and artificial adaptive systems strongly depend on the type of inputs they receive during the developmental process. For example, if kittens are raised in a room with only vertical black and white lines, they forever lose the ability to see horizontal objects [2]. Since active vision, i.e. the sequential and interactive process of selecting and analyzing parts of a visual scene, selects the subset and sequence of images that the visual system perceives, the development of an "healthy" visual system may require free exploration of the visual scene by the animal.

Computational models of developmental visual systems assume that the learning system constructs compact or sparse representations of sensory events that reflect their statistical properties [6], [4], [12], [14] but do not allow the system to freely interact with the environment and choose those sensory events.

Indeed, recent studies suggest that the statistical properties of sensory inputs significantly depend on the behavior of the perceivers [1]. Furthermore, it has been shown that active perception in a naturalistic environment significantly changes the expression of plasticity itself in the rat's cortex [13]. These results suggest that the development of the visual system largely depends not only on the characteristics of the visual field but also on the behavior of the perceivers.

In this paper we explore the role of active body movement in the formation of the visual system by studying the development of visual receptive fields and behavior of robots under active and passive movement conditions. We show that the receptive fields and behavior of robots developed under active condition significantly differ from those developed under passive condition. Our analyses show that the coherence of receptive fields developed in active condition plays an important role in the performance of the robot.

\section{Methods}

The neural control system of a mobile robot equipped with a pan/tilt camera is evolved by means of a genetic algorithm to perform collision-free navigation in an enclosed space using only visual information (figure 1). The evolutionary algorithm evaluates each neural controllers with random mutations until an evolutionary stable control strategy is found [11]. The visual receptive fields of the neural network are modified online while the robot is evaluated. The modification consists of an Hebbian learning rule that tends towards the principal components of the input image set. At the end of the evaluation phase, the resulting receptive fields are not memorized in the genotype of the neural controller. The interactions between evolution and learning are described in a separate paper [5]. In this paper we study the development of receptive fields in evolved robots, that is in robots that are capable of displaying collision-free navigation. In particular, we analyze 

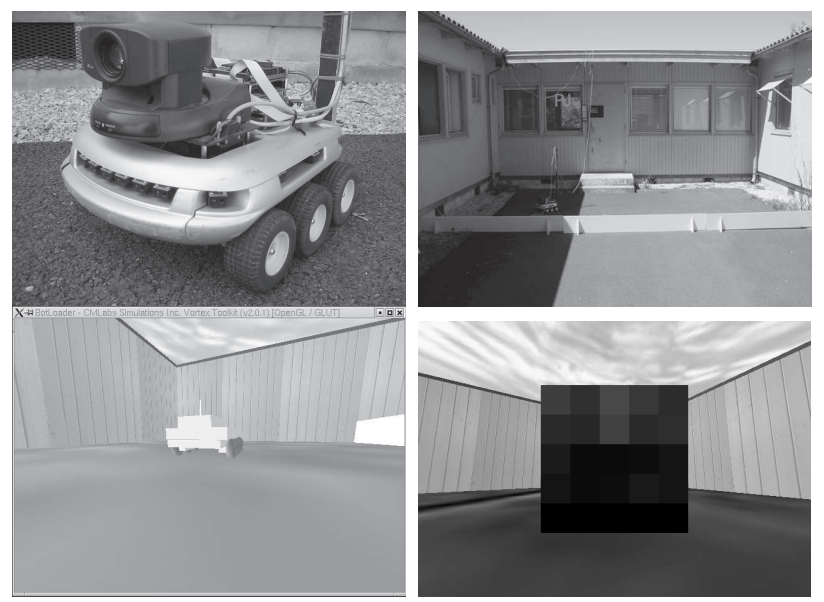

Fig. 1. Upper left: The Koala mobile robot by K-Team ${ }^{T M}$ with pan/tilt camera by Sony ${ }^{T M}$. Upper right: The real environment. Lower left: Simulation of the robot and the environment. Lower right: View from the simulated camera. The robot is capable of visually accessing the $5 \times 5$ pixels in the center of the image.

the formation of receptive fields while the robot is let free to behave according to the evolved sensory motor pathways and while the robot is constrained to move according to a variety of externally imposed motor commands. We call the first case "active behavior" and the second "passive behavior" in accordance to the methodology used in the kittens study. In order to collect data from several independent runs and perform rigorous statistical analysis, we used fast, physicsbased simulations of the robot and its environment (figure 1).

We simulated the robot and the environment using Vortex libraries, a commercially available software package that models gravity, mass, friction, and collisions (http://www.cm-labs.com). The texture of the surfaces in the simulated environment was generated from pictures taken in the real outdoor environment. The robot has six wheels, but only the central wheel on each side is motorized. The pan and tilt angles of the camera are controlled by two separate and independent motors.

The neural network is characterized by a feedforward architecture with evolvable thresholds and discrete-time, fullyrecurrent connections at the output layer (figure 2). A set of visual neurons, arranged on a grid, with non-overlapping receptive fields receives information about the gray level of the corresponding pixels in the image provided by the camera on the robot. The receptive field of each neuron covers a square area of 48 by 48 pixels in the image. We can think of the total area spanned by all receptive fields ( 240 by 240 pixels) as the surface of an artificial retina. The activation of a visual neuron, scaled between 0 and 1 , is given by the average gray level of all pixels spanned by its own receptive field or by the gray level of a single pixel located within the receptive field. The choice between these two activation methods, or filtering strategies, can be dynamically changed by one output neuron at each time step. Two proprioceptive neurons provide input information about the measured horizontal (pan) and vertical (tilt) angles

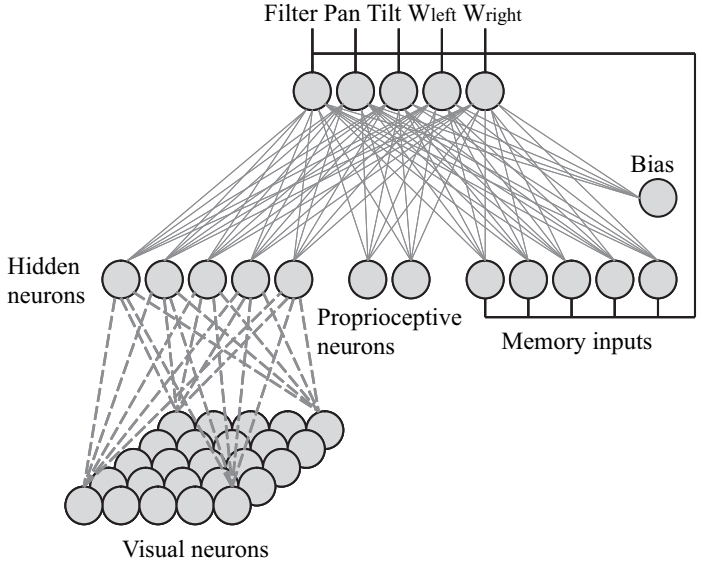

Fig. 2. The architecture is composed of a grid of visual neurons with nonoverlapping receptive fields whose activation is given by the gray level of the corresponding pixels in the image; a set of proprioceptive neurons that provide information about the movement of the camera with respect to the chassis of the robot; a set of output neurons that determine at each sensory motor cycle the filtering used by visual neurons, the new pan and tilt speeds of the camera, and the rotational speeds of the two wheels of the robot; a set of memory units whose outgoing connection strengths represent recurrent connections among output units; and a bias neuron whose outgoing connection weights represent the thresholds of the output neurons.

of the camera. These values are in the interval $[-100,100]$ and $[-25,25]$ degrees for pan and tilt, respectively. Each value is scaled in the interval $[0,1]$ so that activation 0.5 corresponds to 0 degrees (camera pointing forward parallel to the floor). A set of memory units store the values of the output neurons at the previous sensory motor cycle step and send them back to the output units through a set of connections, which effectively act as recurrent connections among output units [3]. The bias unit has a constant value of -1 and its outgoing connections represent the adaptive thresholds of output neurons [8, ch. 5].

Hidden and output neurons use the sigmoid activation function $f(x)=1 /(1+\exp (-x))$ in the range $[0,1]$, where $x$ is the weighted sum of all inputs. Output neurons encode the motor commands of the active vision system and of the robot for each sensory motor cycle. One neuron determines the filtering strategy used to set the activation values of visual neurons for the next sensory motor cycle. Two neurons control the movement of the camera, encoded as speeds relative to the current position. The remaining two neurons encode the direction and rotational speeds of the left and right motored wheels of the robot. Activation values above - and below- 0.5 stand for forward—and backward—rotational speeds.

The connection strengths between visual neurons and hidden neurons are modified by means of an Hebbian learning rule, which has been shown to produce connection strengths that approximate the eigenvectors corresponding to the principal eigenvalues of the correlation matrix of the input patterns [15]. In other words, this learning rule approximates Principal Component Analysis of the input images [10]. The modification of connection strength $\Delta w_{i j}$ depends solely on postsynaptic and presynaptic neuron activations $y_{i}, x_{j}$, 


$$
\Delta w_{i, j}=y_{i}\left(x_{j}-\sum_{k=1}^{i} w_{k j} y_{k}\right)
$$

where $k$ is a counter that points to postsynaptic neurons up to the neuron whose weights are being considered. The new connection strengths are given by $w^{t+1}=w^{t}+\eta \Delta w_{i j}^{t}$ where $0<\eta \leq 1$ is the learning rate, which in these experiments starts at 1.0 and is halved every 80 sensory motor cycles. This learning rule has been used in previous computational models of receptive field development [6] and is intended to capture a system-level property of visual plasticity, not the precise way in which biological synaptic strengths are modified in the visual cortex. Among the several available models of synaptic plasticity [9], we opted for this one because it can be applied online while the robot moves in the environment and because it is equivalent to a widely used technique for image compression.

The neural network is updated at discrete time intervals of $300 \mathrm{~ms}$. At each time interval (sensory motor cycle), the following steps are performed: 1 . the activations of the visual and proprioceptive neurons are computed, the values of the memory units are set to the values of the output units at the previous time step (or to zero if the individual starts its "life"); 2. the activations of the hidden units are computed and normalized; 3. the activations of the output units are computed; 4. the camera and wheels of the robot are set at the corresponding rotational speed for one sensory motor cycle; 5 . the connection weights from visual neurons to hidden neurons are modified using the current neuron activation values.

In step 2 the activations of five hidden units are normalized to operate within the same range of variability in order to equalize the contributions of hidden units to activations of the output units. We emphasize that this procedure is necessary to prevent the output units of the neural network to depend mainly on the activation of the first one or two hidden units. Our preliminary study shows that the principal five eigenvalues of the correlation matrix of the input vector corresponding to the variances of activation of the hidden neurons have different magnitudes, as shown in figure 3 . This means that the activations of the hidden neurons will display different ranges of variability. The normalized output value of the $k$ th hidden neuron $o_{k}^{\prime}$ is computed by: $o_{k}^{\prime}=o_{k} \times s_{1} / s_{k}$ where $o_{k}$ and $s_{k}$ denote the current output value of $k$ th hidden neuron and the standard deviation of all stored output values up to the current time step $\left(s_{1}\right.$ when $\left.k=1\right)$.

The connections between visual neurons and hidden neurons are randomly initialized at the beginning of the life of each individual. The neural network has 65 evolvable connections that are individually encoded on five bits in the genetic string (total length=325). A population of $n$ genomes is randomly initialized by the computer. Each individual genome is then decoded into the connection weights of the neural network (except for the connections from visual neurons to hidden neurons, which are randomly initialized) and tested on the robot while its fitness is computed. The best $20 \%$ individuals

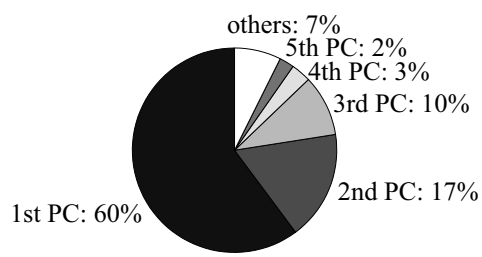

Fig. 3. Percentage of eigenvalues of the first five principal components of the visual input as portions of summation of all 25 eigenvalues. The input vector is composed of the images uniformly sampled in the environment.

(those with highest fitness values) are reproduced, while the remaining $80 \%$ are discarded, by making an equal number of copies so to create a new population of the same size. These new genomes are randomly paired, crossed over with probability 0.1 per pair, and mutated with probability 0.01 per bit. Crossover consists in swapping genetic material between two strings around a randomly chosen point. Mutation consists in toggling the value of a bit. Finally, a copy of the best genome of the previous generation is inserted in the new population at the place of a randomly chosen genome (elitism) in order to improve the stability of the evolutionary process.

\section{SIMULATIONS AND RESULTS}

\section{A. Evolution}

The fitness function was designed to select robots for their ability to move straight forward as long as possible for the duration of life of the individual. This corresponds to the amount of forward rotation of the two motorized wheels of the robot. Each individual is decoded and tested for four trials, each trial lasting 400 sensory motor cycles ${ }^{1}$. A trial can be truncated earlier if the operating system detects an imminent collision by using infrared distance sensors positioned around the body of the robot. The fitness criterion $F\left(S_{\text {left }}, S_{\text {right }}\right)$ is a function of the measured speeds of the left $S_{\text {left }}$ and right $S_{\text {right }}$ wheels:

$$
\begin{gathered}
F\left(S_{\text {left }}, S_{\text {right }}\right)=\frac{1}{E \times T} \sum_{e=0}^{E} \sum_{t=0}^{T^{\prime}} f\left(S_{\text {left }}, S_{\text {right }}, t\right) \\
f\left(S_{\text {left }}, S_{\text {right }}, t\right) \\
=\left(S_{\text {left }}^{t}+S_{\text {right }}^{t}\right) \times\left(1-\sqrt{\frac{\left|S_{\text {left }}^{t}-S_{\text {right }}^{t}\right|}{2 \times S_{\text {max }}}}\right)
\end{gathered}
$$

where $S_{\text {left }}$ and $S_{\text {right }}$ are in the range $[-8,8] \mathrm{cm} / \mathrm{sec}$ and $f\left(S_{\text {left }}, S_{\text {right }}, t\right)=0$ if $S_{\text {left }}$ or $S_{\text {right }}$ is smaller than 0 (backward rotation); $E$ is the number of trials (four in these experiments), $T$ is the maximum number of sensory motor cycles per trial (400 in these experiments), $T^{\prime}$ is the observed number of sensory motor cycles (for example, 34 for a robot whose trial is truncated after 34 steps to prevent collision with a wall). At the beginning of each trial the position

\footnotetext{
${ }^{1}$ Preliminary experiments reported in [5] show that less than 300 updates are necessary to stabilize the plastic weights from visual to hidden neurons.
} 
and orientation of the robot are instantly randomized and the synaptic weight values are re-initialized to random values. We performed these replications of the evolutionary run starting with different genetic populations. In all cases the fitness reached stable values in less than 20 generations (figure 4) which corresponded to collision-free trajectories. Notice that the fitness can never be one because the robot must rotate in order to avoid walls.

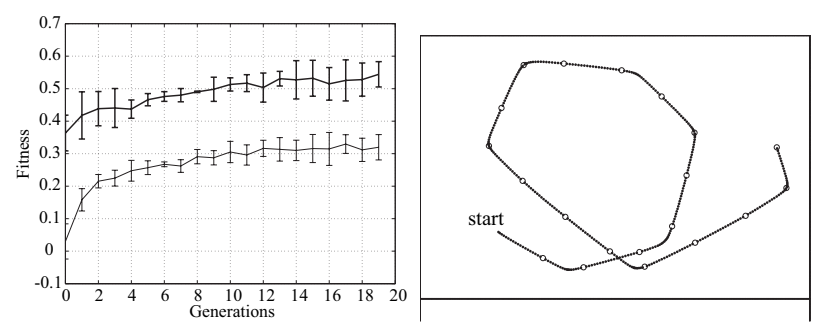

Fig. 4. Evolution with synaptic plasticity. Left: Population average (thin line) and best fitness (thick line) during evolution in physics-based simulations. Each data point is the average of three evolutionary runs with different initializations of the population. Right: An example of trajectory of the best individual in the last generation while synaptic plasticity is active. A dot is plotted every 20 sensory motor cycles. Vertical lines show the standard error.

\section{B. Development under two behavioral conditions}

We studied the development of receptive fields and behavior of the best evolved individuals in two conditions: one where the evolved robot was free to control the movements of its wheeled platform and of the camera, and another where the movement of the wheeled platform was constrained (but not that of the camera). First, we let the evolved robot move freely while the receptive fields were developed (we label the resulting receptive fields in active movement condition $R F_{a}$ ). In the second condition, the same evolved robot was constrained to move according to four pairs $\left(S_{\text {left }}, S_{\text {right }}\right)$ of wheel speeds while the receptive fields were developed.

- Behavior 1: $\left(S_{\text {left }}, S_{\text {right }}\right)=\left(S_{\max },-S_{\max }\right)$

- Behavior 2: $\left(S_{\text {left }}, S_{\text {right }}\right)=\left(0.4 \times S_{\max },-0.4 \times S_{\max }\right)$

- Behavior 3: $\left(S_{\text {left }}, S_{\text {right }}\right)=\left(S_{\text {max }}, 0\right)$

- Behavior 4: $\left(S_{\text {left }}, S_{\text {right }}\right)=\left(S_{\max }, 0.2 \times S_{\max }\right)$

where $S_{\max }$ denotes the maximum speed of the wheels ( 8 $\mathrm{cm} / \mathrm{s}$ ). We call these four behaviors "passive" to highlight that the evolved neural network can not control the wheels ${ }^{2}$ and label the resulting receptive fields $R F_{p 1}, R F_{p 2}, R F_{p 3}$, and $R F_{p 4}$. Behavior 1 and 2 correspond to 'turning-on-the-spot' while behavior 3 and 4 produce small circular behaviors with different radii. The camera could be freely controlled by the evolved neural controller in all four passive conditions.

\footnotetext{
${ }^{2}$ Passive behavior was accomplished by simply neglecting the output values $\left(W_{\text {left }}, W_{\text {right }}\right)$ of the neural controller and reading one of the four pairs $\left(S_{\text {left }}, S_{\text {right }}\right)$ of wheel speeds instead. However note that the output values $\left(W_{\text {left }}, W_{\text {right }}\right)$ were not overwritten by $\left(S_{\text {left }}, S_{\text {right }}\right)$ but copied to the memory units so that passive behavior of the robot would be analogous to that of the kitten carried in a gondola in that they could move their wheels or legs freely without any contribution to the actual movement of their whole bodies.
}

The robot was located randomly at the beginning of each test and allowed to move for 400 sensory motor cycles while the visual receptive fields were developed from initial random weights. The test was repeated ten times for each condition starting from different random weights and locations.

After development in the active and four passive conditions the corresponding receptive fields $R F_{a}, R F_{p 1}, R F_{p 2}, R F_{p 3}$ and $R F_{p 4}$ were fixed and the performance of the robot was evaluated while the robot moved freely for maximum 400 sensory motor cycles.

Figure 5 shows the receptive fields resulting from active and passive behaviors of one trial. Figure 6 shows the result of the performance tests in the five conditions. The performance obtained with receptive fields developed during active behavior $\left(R F_{a}\right)$ is significantly better than those with receptive fields developed during passive behavior $\left(R F_{p 1-4}\right)$.

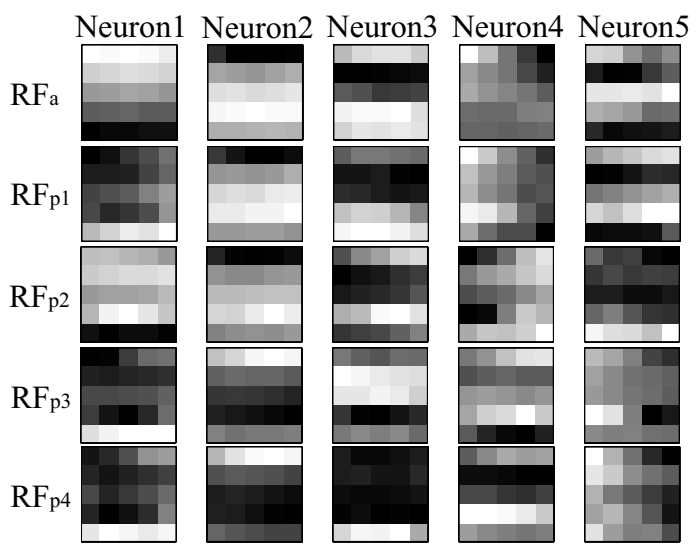

Fig. 5. Receptive fields of five hidden neurons developed in active and passive conditions. Small shaded squares represent the connection strengths from visual neurons, scaled so to fill the gray scale from black (minimum value) to white (maximum value). The leftmost receptive field in each row corresponds to the first principal component of the visual input experienced by the robot. A receptive field is the pattern of synaptic strengths to a neuron, here plotted as a gray level matrix.

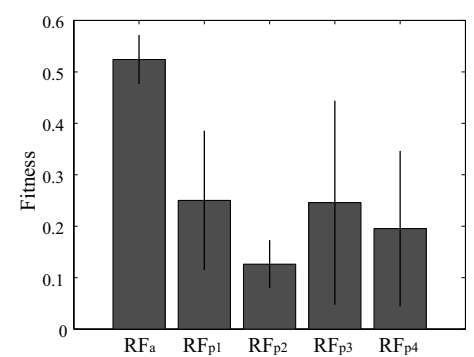

Fig. 6. Performances of the robot with receptive fields developed in active $\left(R F_{a}\right)$ and passive $\left(R F_{p 1-4}\right)$ conditions. The fitness values are averaged over ten tests. Vertical lines show the standard error.

\section{ANALYSIS}

\section{A. Lesion Studies}

The objective of this section is to understand why the performance of $R F_{a}$ differs from that of $R F_{p 1-4}$. We investigated 
the role of $R F_{a}$ by lesioning hidden units one at a time and testing the lesioned controller in the environment ten times for a duration of 400 sensory motor cycles each. Lesion was performed by clamping the activation value of the neuron to a constant value of 0.5 (approximately equal to the average activation level). During these tests the receptive fields were not allowed to change.

Figure 7 shows that lesions of the first and second units (units 1-2) affects performance most significantly in the case of $R F_{a}$. This finding was validated by another set of tests where simultaneous lesion of the first two units significantly reduced the robot's performance, but simultaneous lesion of the last three units did not.

These results can not be simply explained by the larger variance attributed to the first two units by the learning algorithm because, as described in section II, the magnitudes of the output of the five hidden units are normalized so that each hidden unit can equally contribute to firing of the output units.

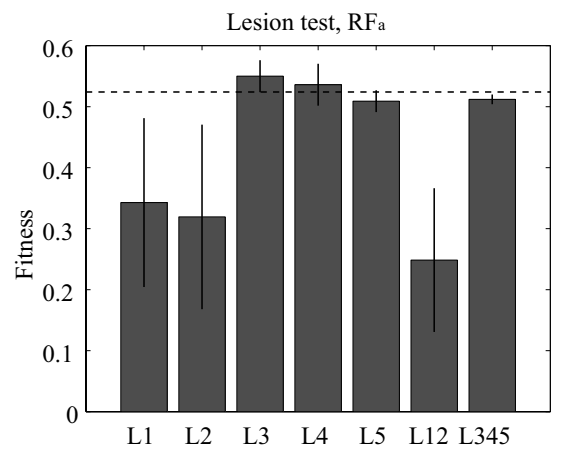

Fig. 7. Performance with lesioned $R F_{a}$. 'L1' denotes the performance of the robot when the first hidden neuron was lesioned; 'L345' when units 3, 4, and 5 were lesioned simultaneously. Fitness values are averaged over ten tests. This figure shows that the first and second hidden neurons play an important role for the performance of the robot. Horizontal dashed line represents the fitness value of the robot with intact receptive fields.

Furthermore, the receptive fields of the first two units developed in passive condition $2\left(R F_{2}\right)$ are similar to those developed in active condition, but the performance of that neural controller is one of the worst observed. A possible explanation of the performance difference between neural controllers developed in active and passive conditions is that the neurons that capture statistically less dominant features (neurons 3, 4, and 5) may develop sensitivity to "interfering" features in the passive conditions. To investigate this hypothesis, we lesioned simultaneously neurons 3,4 , and 5 in the passive conditions and tested the performance of the robot. Figure 8 shows that the performances of the robot were, as expected, improved by lesioning units 3, 4, and 5. These neurons may interfere with the first two neurons by capturing information that "distracts" or contrasts the information provided by the first two neurons, which encode statistically dominant features of the environment.

If the coherence of the receptive fields at least as important as the actual information encoded, then substituting receptive field developed in passive condition with those developed in active condition should not restore the performance of the robot fully. In a first set of tests, the receptive fields of units 3, 4, and 5 of neural controller developed in passive conditions were substituted by those developed in active condition (figure 9, gray bars). The performances of the robot with modified $R F_{p 1-4}$ were not consistently better as when lesioning units 3, 4, and 5. A notable exception is the case of $R F_{p 2}$. The performance is very close to that with $R F_{a}$ because the receptive fields of the first two units are very similar. In the second set of tests, the substitution concerned the receptive fields of the first two units (figure 9, white bars). Also in this case, the performance of the robot was not as good as that obtained by the neural controller developed in active condition.

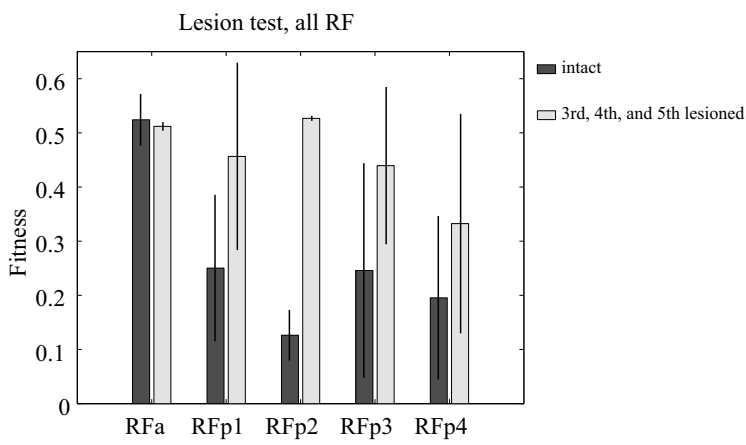

Fig. 8. Performance with lesioned receptive fields. Dark gray bar shows the performance with five intact receptive fields, whereas light gray bar with three lesioned neurons. The fitness values are averaged over ten tests. The performances with all of $\mathrm{RF}_{p 1-4}$ were improved by lesioning units 3 , 4, and 5.

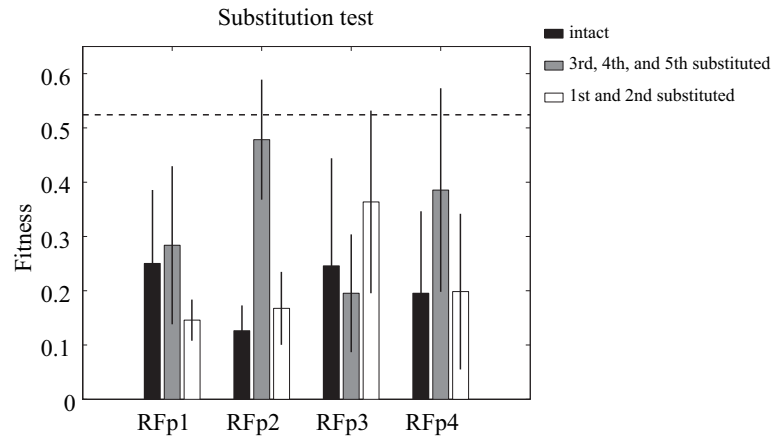

Fig. 9. Performance in the substitution test. Fitness value of the robot was computed when units 3,4 , and 5 of $R F_{p 1-4}$ were substituted by those of $R F_{a}$ (gray bars) and when units 1 and 2 of $R F_{p 1-4}$ were substituted by those of $R F_{a}$ (white bars). Black bar shows the performance with five intact receptive fields for the sake of comparison. Horizontal dashed line represents the fitness value of the robot with intact $R F_{a}$. The fitness values are averaged over ten tests.

\section{DISCUSSION}

The present results suggest that constraints on body movement disturb the development of "healthy" visual receptive 
fields. Although we can not see any significant difference in the level of receptive fields themselves, they caused a significant difference in behavior. Furthermore we have shown that the coherence of receptive fields developed in active condition plays an important role in the good performance of the robot.

Although the arrangement and relative importance of the receptive fields described depend on the specific learning rule used in these experiments, the results suggest that during passive movement the developing system incorporates sensory stimulation that is not functional for normal behavior. In other words, freely behaving systems select a subset of stimuli that coherently support the generation of behavior itself.

One would say that we could conceivably have evolved a robot that would also produce correct behavior under conditions p1-4 if these were presented during evolution, and thus we have only demonstrated that the robot is not good at doing something which it was not evolved to do. This criticism would miss the point of the study which is to demonstrate how motor activity affects development. Evolution is free to pick up a convenient pattern of motor activity that facilitates development. If it were easier to ignore motor activity and perform some sort of non-historical image analysis on every visual input so as to extract the necessary information for navigation, evolution would have very likely found that solution or something close, but that is not the case.

It would be good to come back here to the bigger picture that was set at the start of the paper: the point that not only visuallyguided behavior depends non-trivially on motor activity (active vision) but that its development relies on it as well. This thesis has a stronger and a weaker version. The weaker says: to the extent that sensory input is dependent on movement, and the development of receptive fields dependent on sensory input, then this development also depends on movement. If you change the pattern of allowed movements, you will affect development. This is what the present experiments have shown in this paper.

However there is a stronger version that includes the former but adds the following: there is also a direct dependence of development on how movement is registered by the system, i.e. on proprioceptive activity, or efferent copies or similar mechanisms for distinguishing self-generated movement from non-self-generated movement. For this stronger version, even if one manages to replicate the precise sensory input (thus removing this indirect dependence on movement), development will also be impaired, because it lacks another fundamental component, the information of how visual input and movement (through proprioception) are coordinated.

This stronger version is what the original kittens' experiment demonstrated in [7]. If we accept that the device effectively "copies" the active kitten's sensory input into the passive kitten's, then the latter's lack of visual development can only be attributed to its lack of the temporal correlation between a measure of actual body movement and the corresponding proprioceptive input (barring other factors such as stress, etc). This situation is not quite the same as the one currently repro- duced with the robot as there is only camera proprioception. To support this stronger version of the argument, one should carry out further experiments with an extended sensory system measuring actual body movement by means of accelerometers or gyroscope.

\section{CONCLUSIONS}

We studied the development of receptive fields and behavior of the evolved robots in active and passive conditions. The neural architecture we adopted for visual feature extraction was a simple feedforward neural network performing PCA. The main contribution of this paper can be summarized in the following two points; 1 . We have shown that the receptive fields and performance of the robot developed in active condition significantly differ from those developed in four passive conditions; 2. A possible explanation of this difference is that the coherence of receptive fields developed in active condition plays an important role in the good performance of the robot. This hypothesis is given support by a set of analyses performed on the neural controller and robot behavior.

\section{ACKNOWLEDGMENTS}

The authors wish to thank Claudio Mattiussi for valuable discussions during the early stages of this work. Thanks also to four anonymous referees for useful comments. MS has been supported by EPFL grant. DF has been supported by the Swiss National Science Foundation, grant no. 620-58049.

\section{REFERENCES}

[1] B. Y. Betsch, W. Einhäuser, K. P. Körding, and P. König. The world from a cat's perspective - statistics of natural videos. Biological Cybernetics, 90:41-50, 2004.

[2] C. Blakemore and G. F. Cooper. Development of the brain depends on the visual environment. Nature, 228:477-478, 1970.

[3] J.L. Elman. Finding structure in time. Congnitive Science, 14:179-211, 1990.

[4] D. Field. What is the goal of sensory coding? Neural Computation, 6:559-601, 1994.

[5] D. Floreano, M. Suzuki, and C. Mattiussi. Active vision and receptive field development in evolutionary robots. Evolutionary Computation, 2004. submitted.

[6] P. J. Hancock, R. J. Baddeley, and L. S. Smith. The principal components of natural images. Network, 3:61-70, 1992.

[7] R. Held and A. Hein. Movement-produced stimulation in the development of visually guided behavior. Journal of Comparative and Physiological Psychology, 56(5):872-876, 1963.

[8] J. Hertz, A. Krogh, and R. G. Palmer. Introduction to the theory of neural computation. Addison-Wesley, Redwood City, CA, 1991.

[9] G. E. Hinton and T. J. (Eds.) Sejnowski. Unsupervised Learning. MIT Press, Cambridge, MA, 1999.

[10] I. T. Jolliffe. Principal Component Analysis. Springer Verlag, New York, 1986.

[11] S. Nolfi and D. Floreano. Evolutionary Robotics: Biology, Intelligence, and Technology of Self-Organizing Machines. MIT Press, Cambridge, MA, 2000.

[12] B. A. Olshausen and D. J. Field. Emergence of simple-cell receptive field properties by learning a sparse code for natural images. Nature, 381:607-609, 1996.

[13] D. B. Polley, C. H. Chen-Bee, and R. D. Fröstig. Two directions of plasticity in the sensory-deprived adult cortex. Neuron, 24:623-637, 1999.

[14] R. Rao and D. Ballard. Predictive coding in the visual cortex: a functional interpretation of some extra-classical receptive-field effects. Nature Neuroscience, 2:79-87, 1999.

[15] T. D. Sanger. Optimal unsupervised learning in a single-layer feedforward neural network. Neural Networks, 2:459-473, 1989. 